\title{
Ocular bobbing with survival
}

\author{
CHARLES CLARKE AND RICHARD STERN
}

From The Middlesex Hospital, London

SYNOPSIS A case is described of prolonged ocular bobbing with survival after subarachnoid haemorrhage from a pontine arteriovenous malformation. Hitherto, this sign has usually been associated with a rapidly fatal outcome.

Ocular bobbing was first described as a rarely observed abnormal eye movement in comatose patients by Fisher (1959). He described repetitive sudden conjugate downward deviation of the eyes with slow return to the resting position. Lateral gaze was invariably abolished in the typical form. None of the patients described by Fisher $(1959,1964)$ or by Nelson and Johnston (1970) recovered from their original illness. Most were comatose and had massive pontine infarction or haemorrhage at necropsy. Further reports (Susac et al., 1970; Boddie, 1972) confirm the sinister implications of this sign and discuss its differentiation from other abnormal eye movements with which it has sometimes been confused.

The present case is of interest because of the relative youth of the patient and because the underlying pathology was unusual. In addition, the patient regained consciousness and recovered sufficiently to leave hospital; the ocular bobbing persisted.

\section{CASE REPORT}

A twenty-four year old right-handed married secretary was admitted as an emergency to The Middlesex Hospital having lost consciousness at work. Witnesses described that she complained of a severe occipital headache for a few seconds before falling to the ground unconscious. There was no relevant previous history and no known hypertension; the patient was not taking an oral contraceptive.

On admission to hospital she was unresponsive to deep pain. Respiration was normal. The fundi were normal. Pupils were $3 \mathrm{~mm}$ in diameter and reacted weakly to light. There was convergence spasm and in

(Accepted 24 June 1975.) the resting position spontaneous rapid conjugate downward deviation of the eyes was observed every two to four seconds with return to the central position after one or two seconds. Some prolonged periods of fixed down gaze were seen which lasted up to a minute. No lateral or vertical eye movements could be elicited on passive head flexion or rotation; these manoeuvres did not alter the ocular bobbing, neither did caloric stimulation with iced water. No abnormal movements of the palate were seen. The limbs were flaccid, the plantar responses weakly flexor. Neck stiffness developed after several hours. Blood pressure was $100 / 60 \mathrm{mmHg}$. Lumbar puncture two hours after admission showed uniformly bloodstained fluid with xanthochromic supernatant. The CSF opening pressure was $280 \mathrm{~mm} \mathrm{CSF}$. Bilateral vertebral and carotid angiography showed an anteriovenous malformation in the anterior twothirds of the pons which also involved the fourth ventricle. It was fed by perforating branches of the basilar artery (Figs 1 and 2).

She began to regain consciousness 24 hours after admission and was alert and able to give an accurate history by the fifth day. Full vertical gaze was regained by the fourteenth day; ocular bobbing and absent lateral gaze persisted. She complained initially of oscillopsia. Caloric responses remained absent bilaterally. Bilateral facial weakness was noted and she was markedly dysarthric. Voluntary palatal movement was impaired but recovered by the third week. In the limbs there was minor 'pyramidal' weakness and incoordination of the right arm and leg. At discharge from hospital in the sixth week she was able to walk with a frame, feed, and dress herself. Ocular bobbing persisted and was present when last seen six months after the presenting haemorrhage.

\section{DISCUSSION}

In our patient, as in previous cases, the selective 

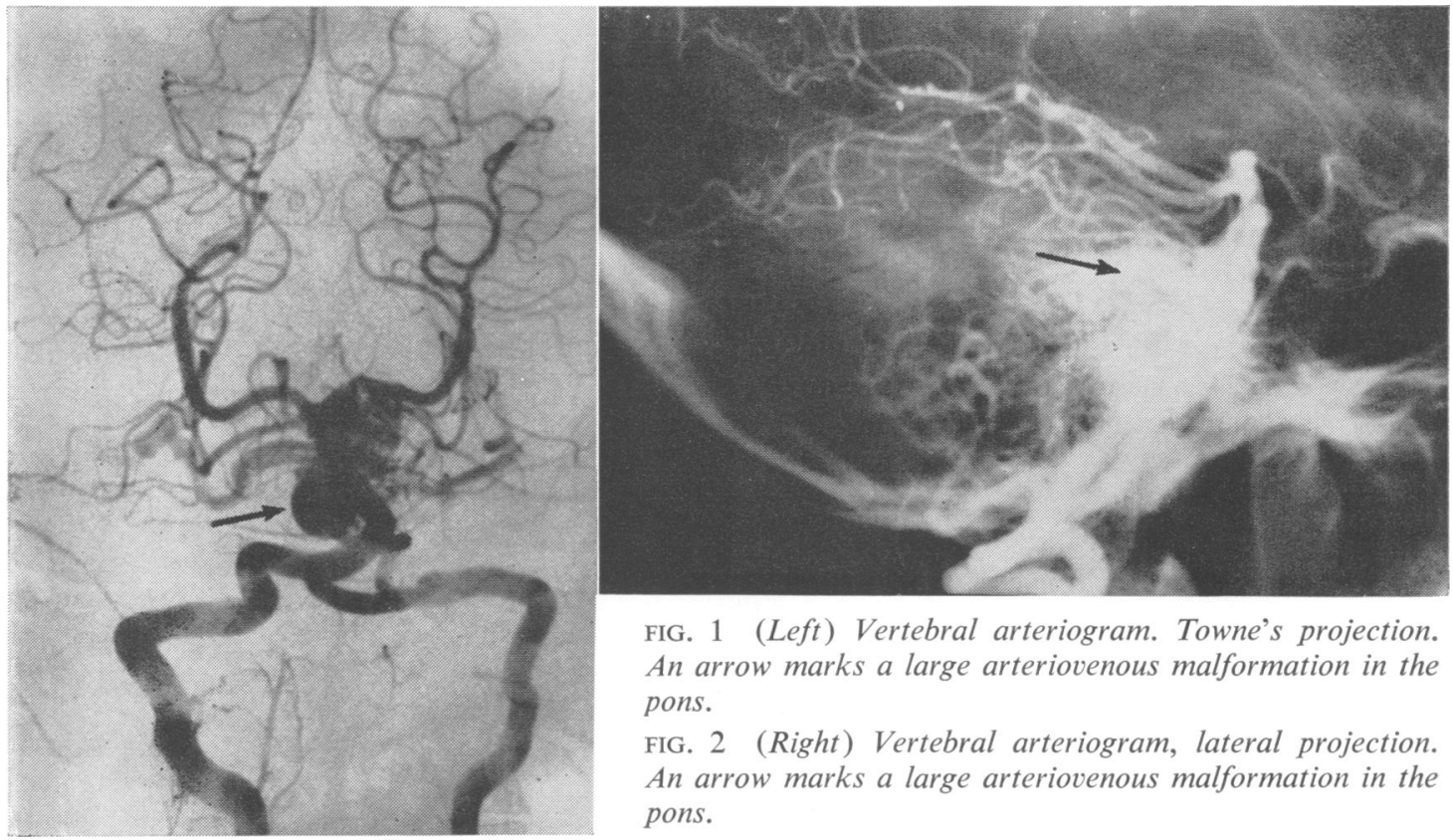

FIG. 1 (Left) Vertebral arteriogram. Towne's projection. An arrow marks a large arteriovenous malformation in the pons.

FIG. 2 (Right) Vertebral arteriogram, lateral projection. An arrow marks a large arteriovenous malformation in the pons.

impairment of conjugate horizontal gaze with preservation of involuntary vertical bobbing was a very striking finding. The anatomical basis for this depends upon the separation at mid-brain level of the descending pathways for vertical and horizontal gaze. The descending pathways for vertical gaze are thought to end at, or near, the oculomotor and trochlear nuclei, whereas those involved in horizontal gaze are thought to end at, or near, the abducens nuclei. Adduction in conjugate lateral gaze is by ascending impulses in a medial longitudinal fasciculus alone without a direct cortical pathway (Walsh and Hoyt, 1969; Bird and Sanders, 1970).

The importance of typical ocular bobbing as a clinical sign in a patient with brain-stem signs is that it is probably evidence of primary pontine damage rather than a cerebellar lesion; it is of particular relevance in the differential diagnosis of a suspected cerebellar haemorrhage. This is well seen in the present case where early angiography confirmed an intrapontine lesion.

Survival of patients showing typical ocular bobbing is the exception (Hameroff et al., 1969; Susac et al., 1970; Boddie, 1972). These authors described three cases who recovered sufficiently to leave hospital. Persistent monocular bobbing was noted (Susac et al. (1970), case 7) in one patient two months after a suspected pontine infarction; transient bobbing (Hameroff et al. (1969), case 5) occurred after myocardial infarction and in the case described by Boddie (hypertensive haemorrhage, presumed pontine) bobbing was replaced by opsoclonus on the fifth day. The present case adds to the clinical spectrum of this abnormal eye movement and is unusual in that the patient not only survived the initial haemorrhage but long after it she was noted to have typical ocular bobbing.

We wish to thank Dr P. Cotton for permission to study the patient under his care, and Professor R. W. Gilliatt, Dr M. J. G. Harrison, and Dr B. Kendall for their help in preparation of this text.

\section{REFERENCES}

Bird, A. C., and Sanders, M. D. (1970). Defects in supranuclear control of horizontal eye movements. Transactions of the Ophthalmological Society of the United Kingdom, 90, 417-432.

Boddie, H. G. (1972). Ocular bobbing and opsoclonus. Journal of Neurology, Neurosurgery, and Psychiatry, 35, 739-742.

Fisher, C. M. (1959). Pathogenesis and Treatment of Cerebro- 
vascular Disease. Edited by W. S. Fields. Thomas: Springfield Ill.

Fisher, C. M. (1964). Ocular bobbing. Archives of Neurology, 11, 543-546.

Hameroff, S. B., Garcia-Mullin, R., and Eckholdt, J. (1969). Ocular bobbing. Archives of Ophthalmology, 82, 774-780.
Nelson, J. R., and Johnston, C. H. (1970). Ocular bobbing. Archives of Neurology, 22, 348-356.

Susac, J. O., Hoyt, W. F., Daroff, R. B., and Lawrence, W. (1970). Clinical Spectrum of ocular bobbing. Journal of Neurology, Neurosurgery, and Psychiatry, 33, 771-775.

Walsh, F. B., and Hoyt, W. F. (1969). Clinical Neuroophthalmology, 3rd edn. Williams and Wilkins: Baltimore. 